\title{
Dynamics and Robust Control of a New Realizable Chaotic Nonlinear Model
}

\author{
M. Higazy (D), ${ }^{1,2}$ Emad E. Mahmoud (D), ${ }^{1,3}$ E. M. Khalil, ${ }^{1,4}$ S. Abdel-Khalek, ${ }^{1,3}$ \\ S. M. Abo-Dahab, ${ }^{5,6}$ and Hammad Alotaibi ${ }^{1}$ \\ ${ }^{1}$ Department of Mathematics, College of Science, Taif University, P. O. Box 11099, Taif 21944, Saudi Arabia \\ ${ }^{2}$ Department of Physics and Engineering Mathematics, Faculty of Electronic Engineering, Menoufia University, \\ Menouf 32952, Egypt \\ ${ }^{3}$ Department of Mathematics, Faculty of Science, Sohag University, Sohag 82524, Egypt \\ ${ }^{4}$ Department of Mathematics, Faculty of Science, Al-Azhar University, Nasr City, Egypt \\ ${ }^{5}$ Department of Mathematics, Faculty of Science, South Valley University, Qena 83523, Egypt \\ ${ }^{6}$ Department of Computer Science, Faculty of Computers and Information, Luxor University, Luxor, Egypt
}

Correspondence should be addressed to Emad E. Mahmoud; e.mahmoud@tu.edu.sa

Received 9 October 2020; Revised 5 November 2020; Accepted 29 December 2020; Published 16 January 2021

Academic Editor: Heng Liu

Copyright (c) 2021 M. Higazy et al. This is an open access article distributed under the Creative Commons Attribution License, which permits unrestricted use, distribution, and reproduction in any medium, provided the original work is properly cited.

We present a new viable nonlinear chaotic paradigm. This paradigm has four nonlinear terms. The essential features of the new paradigm have been investigated. Our new system is confirmed to have chaotic behaviors by calculating its Lyapunov exponents. The relations of the system states are displayed by a suggested new signal flow graph (SFG). The proposed SFG is discussed via some graph theory tools, and some of its hidden features are calculated. In addition, the system is realized via constructing its electronic circuit which helps in the real applications. Also, a robust controller for the system is designed with the aid of a genetic algorithm.

\section{Introduction}

The first noteworthy autonomous chaotic paradigm was proposed by Lorenz in 1963 to model the dynamics of the atmospheric convection with three connected differential equations [1]. Subsequent, Rossler constructed 3D chaotic paradigm with single cross-product nonlinear term [2]. Another 3D chaotic paradigm called the Chen model was constructed by Chen and Ueta in 1999, and they proved that it is a dual and not equivalent to the Lorenz model [3]. After that, in 2002, Lü, in [4], constructed an important chaotic paradigm between Lorenz and Chen models, and then the generalized Lorenz model [5] was constructed as a link of Lorenz, Chen, and Lü paradigms. Few years ago, a novel 3D chaotic paradigm with complicated chaotic behavior and interesting features was constructed by Qi et al. in [6]. During the last ten years, constructing and studying new chaotic paradigms have attracted many researchers in various fields because the chaos phenomena have been found in several modern applications such as communication algorithms, signal processing, nonlinear networks, and chemical and biological structures. In addition, several real applications come up to several new and interesting research points as chaos control techniques [7, 8] and synchronization of chaos systems $[9,10]$.

The $2 \mathrm{D}$ autonomous systems cannot have chaos features as demonstrated by Poincare-Bendixson theorem [11]. Also, in [12], the authors have proved that $3 \mathrm{D}$ dissipative quadratic models represented by ODEs, with total four terms at the right-hand side, cannot have chaos features. After that, in [13], Sprott suggested one quadratic nonlinear term chaotic model in which the right-hand side contains only five terms. From algebraic point of view, the system is considered the simplest chaotic model. The most interesting challenge is to design a 3D autonomous quadratic chaotic paradigm having complicated attractor entity. So, we hope to introduce a new 
chaotic nonlinear model with seven terms at the right-hand side and four of them are nonlinear.

The following conditions are satisfied by the chaotic models $[14,15]$ : (i) very sensitive to starting conditions; (ii) have a single positive Lyapunov exponent. Due to the high capacity, high security, and high efficiency of dealing with chaotic systems, it has widely potentially utilized in nonlinear circuits, secure communications, lasers, neural networks, biological systems, and so on; then, studying chaotic nonlinear models is quite significant these days $[16,17]$.

Representing the complicated paradigms by their signal flow graph is very helpful. It helps in understanding the structure and the complexity of the paradigm using the tools of the graph theory [18]. In directed graph theory, finding directed cycles in the graph is known as a common source of complexity [19]. Those cycles are significant in the context of engineering structures. Specially, they are significant providers of complexity. For instance, cycles can produce positive feedback loops [20], which drive the system to be unstable. Cycles in engineering paradigms also increase the complexity of design and analyse the context of simulation convergence [19]. There are several studies in the literature on graph complexity criteria [19]. Such criteria can either directly or indirectly be linked to the values of eigenvalues of the studied graph matrices.

In real life and in process control, it is very important to control systems in order to work in a desired stable steady operating situation. All of the following are examples of the real processes that need to operate in a predetermined states, adjusting satellite orbits, controlling missile tracks in military applications, in space applications, and in industry also where it is important to control temperature, pressure, and other process variables at specific operating values. Consequently, track control methods of chaotic paradigms have taken a great attention. In [21], Yang, Chen, and Yau proposed a track controller for Lorenz structure. Their reference values were suggested to obey certain constraints which has restricted the applicability of their controller. In [22], Gao developed a track controller in such a way to avoid the constraints of the method in [21]. In [23], a novel strategy for complete and phase robust synchronizations of chaotic nonlinear systems based on singlestate feedback track synchronization control technique and genetic algorithm was proposed. More studies on control of nonlinear systems can be found in [24-28]. Different control strategies can be found in [29-32].

The contribution and addition to this paper is to present a new chaotic nonlinear mathematical model. This model has applications in engineering and communications, and we will prove this by making an electronic circuit for this model. Here, we follow the same strategy to design a new track controller in order to drive the proposed chaotic system to follow any desired reference points. This control technique is designed using one state variable as a feedback variable which reduces the needed sensors and make it easy to implement in real and also minimize the cost.

The remaining parts of our work are organized as follows: the model description and its basic properties are investigated in five sections in Section 2. The proposed SFG of the system and its discussion are studied in Section 3. Designing the electronic circuit that implements the system is presented in Section 4. The method of singlestate feedback control for the proposed system is presented in Section 5. The robustness of the proposed controller is studied and discussed in Section 6. The conclusion of our study is put at the end of the paper before the list of cited references.

\section{System Characterization and Its Basic Features}

At first, we suggest a novel three-dimensional autonomous paradigm:

$$
\begin{aligned}
& \dot{x}_{1}(t)=a_{1}\left(x_{2}(t)-x_{1}(t)\right)+x_{2}(t) x_{3}(t), \\
& \dot{x}_{2}(t)=a_{2} x_{2}(t)-a_{3} x_{1}(t) x_{3}(t), \\
& \dot{x}_{3}(t)=a_{4} x_{1}^{2}(t)-a_{5} x_{3}(t),
\end{aligned}
$$

where $\left(x_{1}(t), x_{2}(t), x_{3}(t)\right) \in R^{3}$ and $a_{1}, a_{2}, a_{3}, a_{4}, a_{5}$ are real parameters.

System (1) has the following essential dynamical features.

2.1. System (1) Generalized Hamiltonian. Smooth nonlinear system (1) is considered, given in the following formula:

$$
\dot{x}=\alpha(\mathbf{x}) \frac{\partial \mathbf{H}}{\partial \mathbf{x}}+\sigma(\mathbf{x}) \frac{\partial \mathbf{H}}{\partial \mathbf{x}},
$$

where $\mathbf{x}=\left[x_{1}(t), x_{2}(t), x_{3}(t)\right]^{T}, \mathbf{H}(\mathbf{x})$ is smooth energy function and universally positive definite, and $(\partial \mathbf{H} / \partial \mathbf{x})$ is a column gradient vector of $\mathbf{H}(\mathbf{x})$.

By using energy function in quadratic formula, we have

$$
\mathbf{H}(\mathbf{x})=\frac{1}{2} \mathbf{x}^{T} \gamma x,
$$

where $\gamma$ signifies a constant diagonal matrix, which is symmetric and positive definite, with respect to $(\partial \mathbf{H} / \partial \mathbf{x})=\gamma x, \alpha(\mathbf{x})$ is antisymmetric matrix representing the vector field of the workless part, and $\sigma(\mathbf{x})$ is a symmetric matrix, a negative definite, representing the working or nonconservative part of the system:

$$
\begin{aligned}
& \alpha(\mathbf{x})=[-\alpha(\mathbf{x})]^{T}, \\
& \sigma(\mathbf{x})=[\sigma(\mathbf{x})]^{T} .
\end{aligned}
$$

Define $\mathbf{H}(\mathbf{x})$ of the model in (1) as

$$
\begin{aligned}
\mathbf{H}(\mathbf{x}) & =\frac{1}{2}\left[\left(\frac{x_{1}(t)}{-a_{3} x_{3}(t) x_{2}(t)}\right)^{2}+\left(\frac{x_{2}(t)}{-a_{3} x_{3}(t)}\right)^{2}+\left(x_{3}(t)\right)^{2}\right], \\
{\left[\begin{array}{c}
\dot{x}_{1}(t) \\
\dot{x}_{2}(t) \\
\dot{x}_{3}(t)
\end{array}\right] } & =\mathbf{A}\left[\begin{array}{c}
\frac{x_{1}(t)}{-a_{3} x_{3}(t) x_{2}(t)} \\
\frac{x_{2}(t)}{-a_{3} x_{3}} \\
x_{3}(t)
\end{array}\right]+\mathbf{B}\left[\begin{array}{c}
\frac{x_{1}(t)}{-a_{3} x_{3}(t) x_{2}(t)} \\
\frac{x_{2}(t)}{-a_{3} x_{3}} \\
x_{3}(t)
\end{array}\right],
\end{aligned}
$$


where

$$
\begin{gathered}
\mathbf{A}=\left[\begin{array}{ccc}
0 & \frac{1}{2}\left(-a_{3} x_{3}(t)\right)\left(a_{1}+x_{3}(t)\right) & -\frac{1}{2} a_{4} x_{1}(t) x_{2}(t)\left(-a_{3} x_{3}(t)\right) \\
-\frac{1}{2}\left(-a_{3} x_{3}(t)\right)\left(a_{1}+x_{3}(t)\right) & 0 & -\frac{1}{2} a_{3} x_{1}(t) \\
\frac{1}{2} a_{4} x_{1}(t) x_{2}(t)\left(-a_{3} x_{3}(t)\right) & \frac{1}{2} a_{3} x_{1}(t) & 0
\end{array}\right], \\
\mathbf{B}=\left[\begin{array}{ccc}
a_{1} a_{3} x_{3}(t) x_{2}(t) & \frac{1}{2}\left(-a_{3} x_{3}(t)\right)\left(a_{1}+x_{3}(t)\right) & \frac{1}{2} a_{4} x_{1}(t) x_{2}(t)\left(-a_{3} x_{3}(t)\right) \\
\frac{1}{2}\left(-a_{3} x_{3}(t)\right)\left(a_{1}+x_{3}(t)\right) & -a_{2} a_{3} x_{3}(t) & -\frac{1}{2} a_{3} x_{1}(t) \\
\frac{1}{2} a_{4} x_{1}(t) x_{2}(t)\left(-a_{3} x_{3}(t)\right) & -\frac{1}{2} a_{3} x_{1}(t) & -a_{5}
\end{array}\right] .
\end{gathered}
$$

The existence of the Hamilton in model (1) implies its ability to model natural phenomena.

2.2. Invariance and Symmetry of the Proposed Model. For system (1), the transformation $\left(x_{1}(t), x_{2}(t)\right.$, $\left.x_{3}(t)\right) \longrightarrow\left(-x_{1}(t),-x_{2}(t), x_{3}(t)\right)$ implies that the system is invariant.

Then, if $\left(x_{1}(t), x_{2}(t), x_{3}(t)\right)$ is a solution of model (1), then $\left(-x_{1}(t),-x_{2}(t), x_{3}(t)\right)$ is a solution of the same model too.

2.3. Dissipation of Proposed Model (1). The divergence of proposed paradigm (1) is

$$
\begin{aligned}
\nabla \cdot V & =\frac{\partial \dot{x}_{1}(t)}{\partial x_{1}(t)}+\frac{\partial \dot{x}_{2}(t)}{\partial x_{2}(t)}+\frac{\partial \dot{x}_{3}(t)}{\partial x_{3}(t)} \\
& =-a_{1}+a_{2}-a_{5} .
\end{aligned}
$$

Then, proposed chaotic paradigm (1) is dissipative such that

$$
-a_{1}+a_{2}-a_{5}<0
$$

2.4. Fixed Point and Its Stability of Proposed Model (1). Solving the following system of equations leads to the equilibria of system (1):

$$
\begin{aligned}
& 0=a_{1}\left(x_{2}(t)-x_{1}(t)\right)+x_{2}(t) x_{3}(t), \\
& 0=a_{2} x_{2}(t)-a_{3} x_{1}(t) x_{3}(t), \\
& 0=a_{4} x_{1}^{2}(t)-a_{5} x_{3}(t) .
\end{aligned}
$$

System (1) has a trivial fixed point $E_{0}=(0,0,0)$.
In order to examine the stability of $E_{0}$, the Jacobian matrix of proposed model (1) at $E_{0}$ is

$$
J_{E_{0}}=\left(\begin{array}{ccc}
-a_{1} & a_{1} & 0 \\
0 & a_{2} & 0 \\
0 & 0 & -a_{5}
\end{array}\right),
$$

the characteristic equation of $J_{E_{0}}$ is

$$
\left(\lambda+a_{1}\right)\left[\lambda^{2}+\lambda\left(a_{5}-a_{2}\right)-a_{2} a_{5}\right]=0,
$$

and as a result, the characteristic equation of $J_{E_{0}}$ has the following three eigenvalues:

$$
\lambda_{1}=-a_{1}, \lambda^{2}+\lambda\left(a_{5}-a_{2}\right)-a_{2} a_{5}=0 .
$$

By applying Routh-Hurwitz theorem, the trivial fixed point is stable if and only if

$$
a_{1}>0, a_{5}-a_{2}>0 \text {, and }-a_{2} a_{5}>0 \text {. }
$$

So, the constraints making the trivial fixed point stable are

$$
a_{1}>0, a_{5}>a_{2} \text {, and } a_{2} a_{5}<0,
$$

otherwise it is unstable.

2.5. Calculating Lyapunov Exponents for Proposed Model (1). Proposed model (1) can be written in vector notation as

$$
\dot{X}(t)=h(X(t) ; \eta) .
$$

Such that $X(t)=\left[x_{1}(t), x_{2}(t), x_{3}(t)\right]^{T}$ presents the vector of state space, $h=\left[h_{1}, h_{2}, h_{3}\right]^{T}, \eta$ presents the parameters, and $[\ldots]^{T}$ signifies matrix transpose operation. The deviations from the $X(t)$ trajectory are given in the following equation: 


$$
\delta \dot{X}(t)=J_{i j}(X(t) ; \eta) \delta X, \quad i, j=1,2,3,
$$

where $J_{i, j}=\left(\partial h_{i} / \partial x_{j}\right)$ is the Jacobian matrix and takes the following form:

$$
J_{i, j}=\left(\begin{array}{ccc}
-a_{1} & a_{1}+x_{3}(t) & x_{2}(t) \\
-a_{3} x_{3}(t) & a_{2} & -a_{3} x_{3}(t) \\
2 a_{4} x_{1}(t) & 0 & -a_{5}
\end{array}\right) .
$$

The Lyapunov exponents $L_{i}$ of the system are defined by

$$
L_{i}=\lim _{t \rightarrow \infty} \frac{1}{t} \log \frac{\left\|\delta x_{i}(t)\right\|}{\left\|\delta x_{i}(0)\right\|}
$$

Numerically solve equations (14) and (15) simultaneously to find $L_{i}$. Order 4 Runge-Kutta algorithm is utilized to estimate $L_{i}$.

The system parameters are selected as follows: $a_{1}=10$, $a_{2}=5, a_{3}=4, a_{4}=2$, and $a_{5}=3$ where the initial values are chosen as $x_{1}(0)=1, x_{2}(0)=2$, and $x_{3}(0)=3$. The Lyapunov exponents are estimated as follows: $L_{1}=1.01, L_{2}=0$, and $L_{3}=-12.5$.

This implies that our proposed system (1) for this selection of the parameters $a_{1}, a_{2}, a_{3}, a_{4}$, and $a_{5}$ is a chaotic paradigm because it has one positive Lyapunov exponent.

Figure 1 proves that our proposed model (1) is chaotic. Solving system (1) numerically, for specific selections of the parameter values, it is clear that it has chaotic behavior in specific domains where initially nearby trajectories break away exponentially with time. For example, Figure 1(a) displays two such numerically evaluated solutions of (1), where we give only the $\left(t, x_{1}(t)\right)$ plot. The chaotic behavior of those solutions is confirmed from the fact that the two initially nearby orbits break away from each other exponentially at about $t \cong 15$. As shown in Figure 1(b), model (1) has a chaotic attractor. In Figure 1(b), we plot the motion in the 3-dimensional space $\left(x_{1}(t), x_{2}(t), x_{5}(t)\right)$.

In Figure 2, we fix $a_{2}=5, a_{3}=4, a_{4}=2$, and $a_{5}=3$ and vary $a_{1} \in[1,30]$. From Figure 2 , it is clear that system (1) has periodic solution when $a_{1} \in[1,8.4]$. The chaotic attractors appear when $\left.a_{1} \in\right] 8.4,19.3$ ]. If $\left.\left.a_{1} \in\right] 19.3,30\right]$, our system has fixed point solutions. Figure 2 proves that our new system contains chaotic solutions over a wide period $\left.a_{1} \in\right] 8.4,19.3$ ]. As we did with the $a_{1}$ parameter, we did with the rest of the parameters. And because we got almost the same results, we will not display the results for the rest of the parameters.

In Figure 2, we find chaotic attractors, periodic attractors, and fixed point solutions of system (1). To check this, we have solved numerically (16) (using, e.g., Mathematica 7 software) in several cases and excellent agreements are found with the results of Figure 2. For example, choosing $a_{2}=5$, $a_{3}=4, a_{4}=2$, and $a_{5}=3$, with the initial conditions $t_{0}=0$, $x_{1}(0)=1, x_{2}(0)=2$, and $x_{3}(0)=3$, and when $a_{1}=7$, the solution of system (1) has periodic solution trivial fixed point (see Figure 3(a)), chaotic attractor when $a_{1}=10$ as in Figure 3(b). In Figure 3(c), $a_{1}=27$, the solution is fixed point.

\section{System Signal Flow Graph}

Representing the complex systems graphically is useful for understanding the relationship between its components. Our system components are its direct state variables $x_{1}, x_{2}$, and $x_{3}$ in addition to the combined three states $x_{1}^{2}, x_{1} x_{3}$, and $x_{2} x_{3}$.

Each state variable is represented by a vertex in the digraph $\mathscr{G}$ of system (1). The edge $(u, v) \in \mathscr{G}$ iff $(\partial v / \partial u) \neq 0$. Then, the adjacency matrix of $\mathscr{G}$ is an image of the relationships between the system state variables of system (1). Figure 4 shows the proposed graph of the studied system.

The following is the weighted adjacency matrix, $A(\mathscr{G})$, of the system's proposed graph:

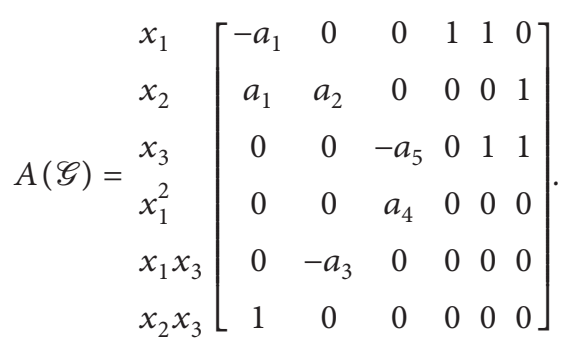

The eigenvalues of the matrix in (9) can be used to compute the energy of the digraph $\mathscr{G}$ (see [19]). To compute the energy of the proposed graph, we follow the method explained in [19] (page 6).

Firstly, formulate the adjacency matrix of the system graph $M$ where

$$
M_{i j}= \begin{cases}1, & \text { for any edge }(u, v), \quad u \neq v \text { of the graph; } \\ 0, & \text { otherwise. }\end{cases}
$$

Then,

$$
M(\mathscr{G})=\begin{gathered}
x_{1} \\
x_{2} \\
x_{3} \\
x_{1}^{2} \\
x_{1} x_{3} \\
x_{2} x_{3}
\end{gathered}\left[\begin{array}{llllll}
0 & 0 & 0 & 1 & 1 & 0 \\
1 & 0 & 0 & 0 & 0 & 1 \\
0 & 0 & 0 & 0 & 1 & 1 \\
0 & 0 & 1 & 0 & 0 & 0 \\
0 & 1 & 0 & 0 & 0 & 0 \\
1 & 0 & 0 & 0 & 0 & 0
\end{array}\right] .
$$

The energy $\mathscr{E}_{\mathscr{G}}$ of the graph $\mathscr{G}$ is calculated via the following formula [19]:

$$
\mathscr{E}_{\mathscr{G}}=\left(\frac{1}{|E|} \sum_{k=1}^{|E|} w_{k}\right) \sum \operatorname{SVD}(M(\mathscr{G}))
$$

where $|E|$ is the number of edges in $\mathscr{G}, w_{|E|}$ is the edge weights, and $\operatorname{SVD}(M(\mathscr{G}))$ is a vector of singular values of matrix. For more details about graph energy, see [19].

Let $\lambda_{1}, \lambda_{2}, \ldots, \lambda_{n}$ are the eigenvalues of the matrix $M^{T} M$, with repetitions. Order these so that $\lambda_{1} \geq \lambda_{2} \geq \cdots \geq \lambda_{n} \geq 0$, assuming $v_{i}=\sqrt{\lambda_{i}}$, then $v_{1} \geq v_{2} \geq \cdots \geq v_{n} \geq 0$, and they are the components of the SVD. Then, 


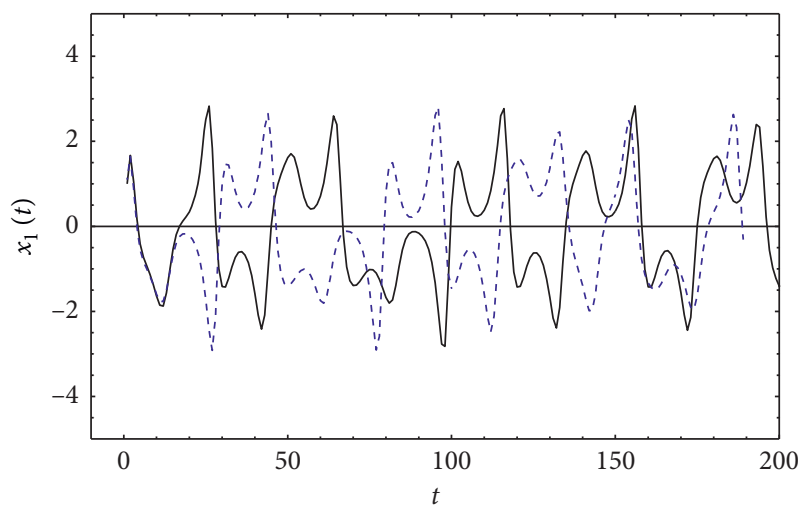

(a)

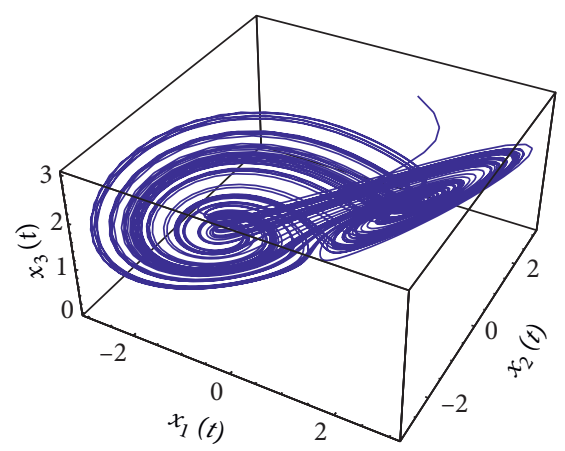

(b)

FIgURe 1: (a) Two numerically calculated solutions of (1) for $a_{1}=10, a_{2}=5, a_{3}=4, a_{4}=2$, and $a_{5}=3$ with $t_{0}=0, x_{1}(0)=1, x_{2}(0)=2$, and $x_{3}(0)=3$ (solid curve) and $x_{1}(0)=1.001, x_{2}(0)=2$, and $x_{3}(0)=3.001$ (dotted curve). Note the exponential separation that becomes clear at $t \cong 15$, clearing the chaotic manner of the system orbits. (b) 3-dimensional chaotic attractor in $\left(x_{1}(t), x_{2}(t), x_{3}(t)\right)$ space.

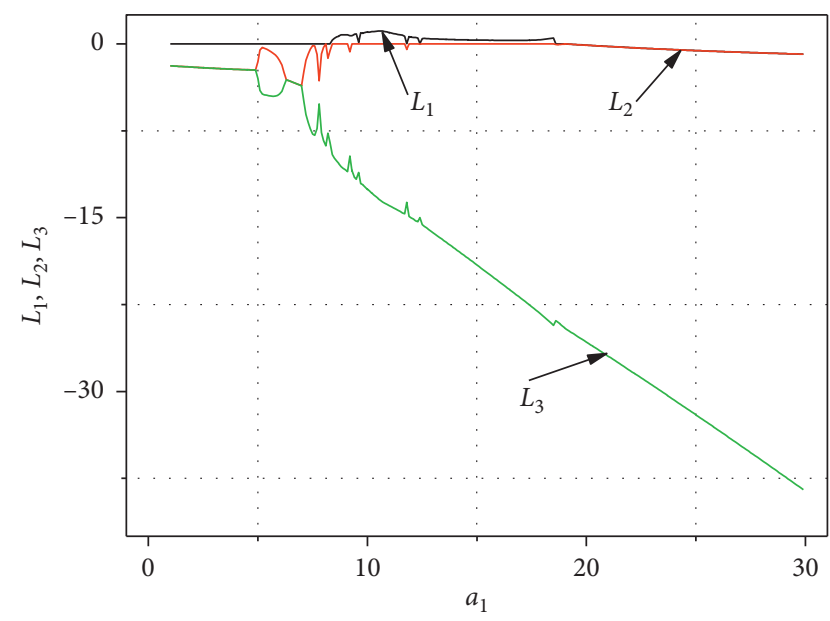

FIGURE 2: $L_{1}, L_{2}$, and $L_{3}$ versus $a_{1}, a_{1} \in[1,30]$ and $a_{2}=5, a_{3}=4$, $a_{4}=2$, and $a_{5}=3$ with the same initial conditions in Figure 1 .

$$
\operatorname{SVD}(M(\mathscr{G}))=[3.5321,2.3473,1.0,1.0,0,12061]
$$

Then, $\mathscr{E}_{\mathscr{G}}=4.5$, that can be used as measure of the system complexity.

\section{System Realization Using NI Multisim}

In this section, we realize the studied system using NI Multisim and its electronic circuit is designed as shown in Figure 5. The circuit consists of three integrators symbolized as X1, X2, and X3 all of which are constructed using the 741 OP. AMP; three summers S1, S2, and A3 each of which has three inputs and one output; two multipliers symbolized with X1X3, X1X1, and X2X3 each of which has two inputs and one output; three 1 micro Farad capacitors C1, C2, and C3 where it is initially charged by 1,2 , and 3 volts, respectively, to realize the initial conditions of the studied system; and ten resistors R1, R2, .., R10, where R1, R3, R5, $\mathrm{R} 7, \mathrm{R} 8, \mathrm{R} 9$, and R10 are equal $10 \mathrm{kohms}$ and R2, R4, and R6 are equal 100 kohms. The input and output gains of the summers are designed as follows (Table 1) in order to realize the studied system. Figure 6 shows the phase portrait of the state variable $x_{2}$ with respect to the state variable $x_{1}$. Figure 7 shows the phase portrait of the state variable $x_{3}$ with respect to the state variable $x_{1}$. Figure 8 shows the phase portrait of the state variable $x_{3}$ with respect to the state variable $x_{2}$.

Table 2 shows the designed values of input/output gains of the used multipliers.

\section{Single-State Feedback Control for the Proposed System}

5.1. Controller Design. In this section, we designed a reference point-based single-state feedback controller. The suggested controller will drive the states of the proposed chaotic model to follow the desired predetermined reference values. The proposed controller's input consists of the output and the states of the chaotic system. The output of the controller is used as an input of the controlled chaotic system.

In [21], the proposed controller was depended on the sliding-mode technique and the Lorenz system states were derived to track the reference values but under some constraints which limit its real applications. In [22], the author proposed a track controller by which the Lorenz system states were derived to follow any of their predetermined reference values. In this work, a reference value single-state feedback controller is to be constructed in such a way that the proposed chaotic model states will be derived to track any desired reference values without any constraints.

Here, it is proposed that the state $x_{2}$ of the studied system is the only state that can be easily measured. For studied system (1), a novel reference point-based single-state feedback controller is designed as follows:

$$
\begin{aligned}
& C_{1}=-a_{1} x_{2 r}+a_{1} x_{1 r}-x_{2 r} x_{3 r}, \\
& C_{2}=k\left(x_{2}-x_{2 r}\right)-a_{2} x_{2 r}+a_{3} x_{1 r} x_{3 r}, \\
& C_{3}=-a_{4} x_{1 r}^{2}+a_{5} x_{3 r},
\end{aligned}
$$




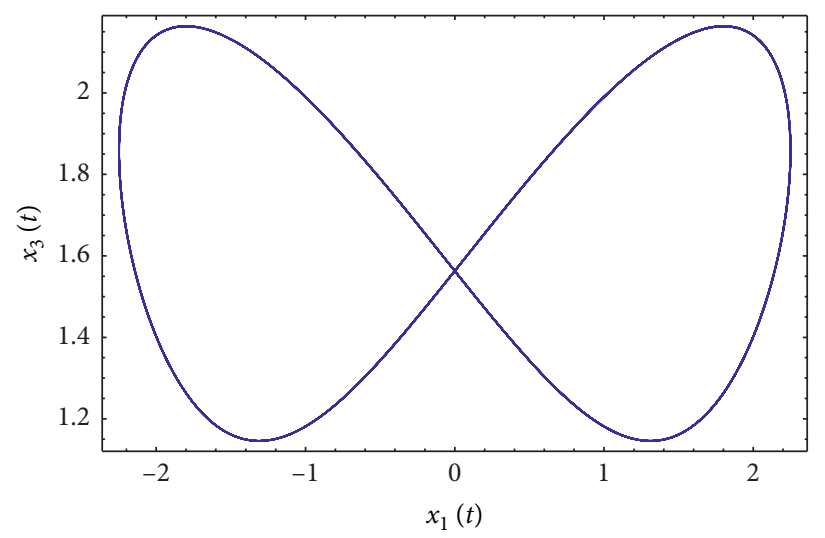

(a)

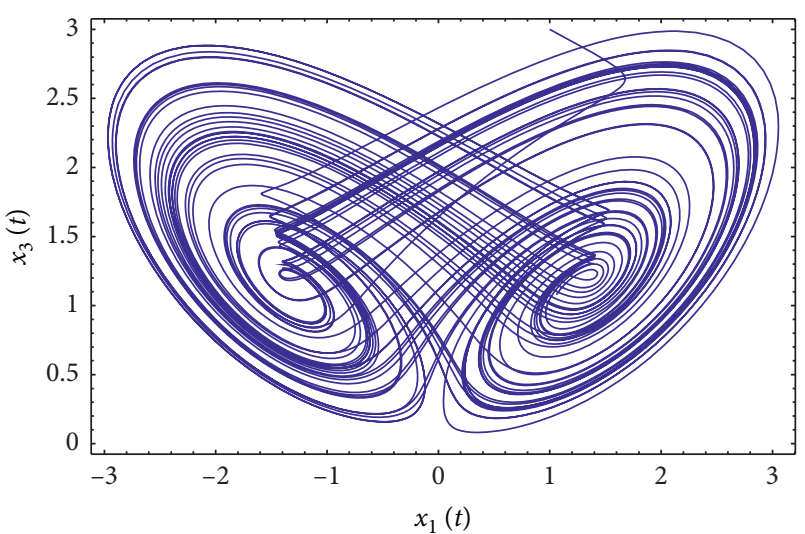

(b)

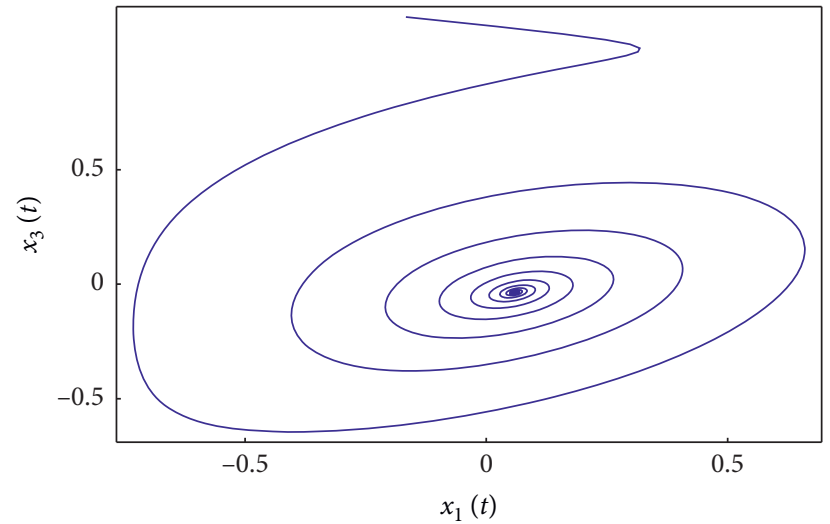

(c)

Figure 3: With the same initial conditions in Figure 1. (a) Periodic solution in $\left(x_{1}(t), x_{3}(t)\right)$ plan, $\mathrm{a}_{1}=7$. (b) Chaotic solution in $\left(x_{1}(t), x_{3}(t)\right)$ plan, $\mathrm{a}_{1}=10$. (c) Fixed point solution in $\left(x_{1}(t), x_{3}(t)\right)$ plan, $\mathrm{a}_{1}=27$.

where $x_{i r}$ is the desired reference value of the system state $x_{i}$ for all $i \in\{1,2,3\}$ and $k$ is the controller gain.

In (24), the closed-loop feedback is constructed only using the second state variable $x_{2}$. Hence, it is called singlestate feedback controller.

The controlled system via the proposed controller becomes

$$
\begin{aligned}
& \left(\frac{\mathrm{d} x_{1}}{\mathrm{~d} t}\right)=a_{1} x_{2}-a_{1} x_{1}+x_{2} x_{3}+C_{1}, \\
& \left(\frac{\mathrm{d} x_{2}}{\mathrm{~d} t}\right)=a_{2} x_{2}-a_{3} x_{1} x_{3}+C_{2}, \\
& \left(\frac{\mathrm{d} x_{3}}{\mathrm{~d} t}\right)=a_{4} x_{1}^{2}-a_{5} x_{3}+C_{3} .
\end{aligned}
$$

Assume that $x_{i r}$ denotes the desired reference value of the state variable $x_{i}$ where $i=1,2,3$. And let $\left\{E_{i}=x_{i}-x_{i r}: i=1,2,3\right\}$ is the errors set between the system state variable and their references. Then, putting $x_{1}=E_{1}+x_{1 r}, x_{2}=E_{2}+x_{2 r}$, and $x_{3}=E_{3}+x_{3 r}$ in (25), the control error dynamical system can be derived as follows:

$$
\begin{aligned}
& \left(\frac{\mathrm{d} E_{1}}{\mathrm{~d} t}\right)=a_{1} E_{2}-a_{1} E_{1}+E_{2} E_{3}+x_{3 r} E_{2}+x_{2 r} E_{3}, \\
& \left(\frac{\mathrm{d} E_{2}}{\mathrm{~d} t}\right)=\left(a_{2}+k\right) E_{2}-a_{3} E_{1} E_{3}-a_{3} x_{3 r} E_{1}-a_{3} x_{1 r} E_{3}, \\
& \left(\frac{\mathrm{d} E_{3}}{\mathrm{~d} t}\right)=a_{4} E_{1}^{2}+2 a_{4} x_{1 r} E_{1}-a_{5} E_{3} .
\end{aligned}
$$

It is clear that the $E_{o}=(0,0,0)$ is an equilibrium of error dynamical system (26) and its Jacobian matrix can be written as

$$
\begin{aligned}
J(E) & =\left[\begin{array}{ccc}
-a_{1} & a_{1}+E_{3}+x_{3 r} & E_{2}+x_{2 r} \\
-a_{3} E_{3}-a_{3} x_{3 r} & \left(a_{2}+k\right) & -a_{3} E_{1}-a_{3} x_{1 r} \\
2 a_{4} E_{1}+2 a_{4} x_{1 r} & 0 & -a_{5}
\end{array}\right], \\
J\left(E_{0}\right) & =\left[\begin{array}{ccc}
-a_{1} & a_{1}+x_{3 r} & x_{2 r} \\
-a_{3} x_{3 r} & \left(a_{2}+k\right) & -a_{3} x_{1 r} \\
2 a_{4} x_{1 r} & 0 & -a_{5}
\end{array}\right] .
\end{aligned}
$$




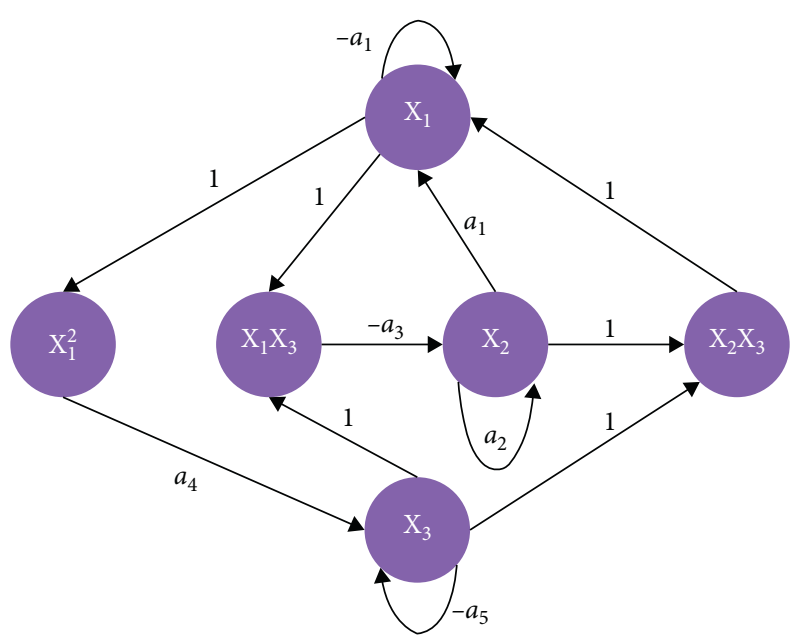

FIgURE 4: Proposed signal flow graph of the proposed chaotic system.

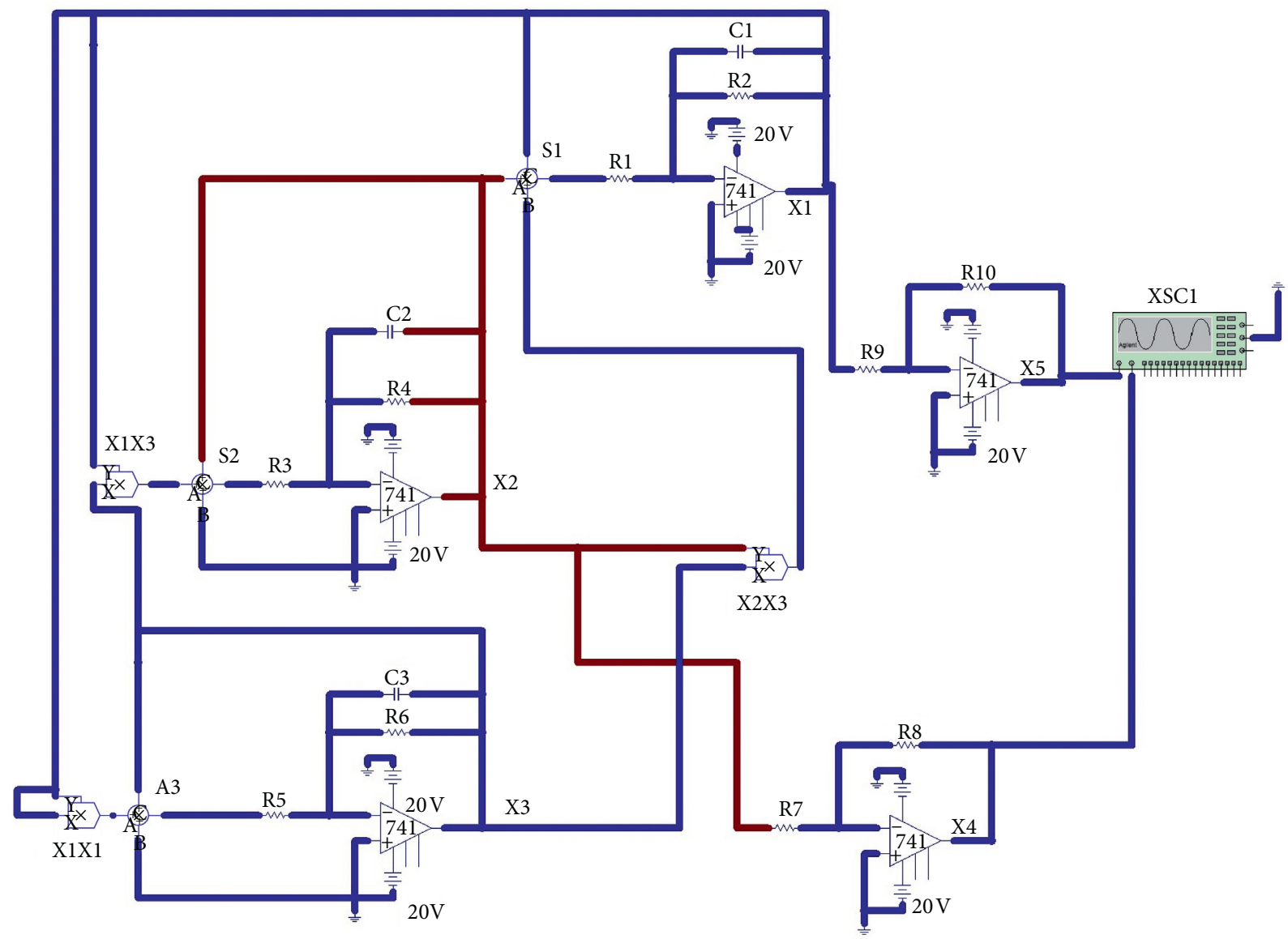

FIgURe 5: The electronic circuit of the proposed system.

If the controller gain $k$ can be calculated such that the eigenvalues of the Jacobian matrix 16 are all stable, the control errors $E_{1}, E_{2}$, and $E_{3}$ will be stable asymptotically at its equilibrium $E_{0}=(0,0,0)$. This imply that the system states $x_{1}, x_{2}$, and $x_{3}$ will follow their desired reference values $x_{1 r}, x_{2 r}$, and $x_{3 r}$, respectively. 
TABLE 1: The input/output gains of the summers.

\begin{tabular}{lcccc}
\hline & Input A gain & Input B gain & Input C gain & Output gain \\
\hline S1 & -10 & 1 & 10 & 1 \\
S2 & -4 & 1 & -5 & 1 \\
A3 & 2 & 0 & 3 & 1 \\
\hline
\end{tabular}

Then, our task is to calculate the suitable value of the gain $k$. Our result is written in the following result.

Theorem 1. Let $\Omega=a_{3} x_{3 r}^{2}+a_{1} a_{3} x_{3 r}-a_{1} a_{2}+a_{1} a_{5}-a_{2} a_{5}$ $-2 a_{4} x_{1 r} x_{2 r}, \quad \Phi=a_{3} a_{5} x_{3 r}^{2}-a_{1} a_{2} a_{5}+2 a_{3} a_{4} x_{3 r} x_{1 r}^{2}+a_{1} a_{3} a_{5}$ $x_{3 r}+2 a_{1} a_{3} a_{4}, \quad a=\left(a_{1}+a_{5}\right), \quad b=\left(-\Omega-a_{1}^{2}-a_{5}^{2}+a_{1} a_{2}-\right.$ $\left.a_{1} a_{5}+a_{2} a_{5}-2 a_{4} x_{1 r} x_{2 r}\right)$, and $c=\left(\Omega a_{5}+\Omega a_{1}-\Phi-\Omega a_{2}\right)$. The states of studied system (1) can follow any desired predetermined values $x_{1 r}, x_{2 r}$, and $x_{3 r}$ via the proposed controller 12 , if the controller gain $k$ is adjusted such that

$$
k<\min \left\{\left(a_{1}-a_{2}+a_{5}\right), \frac{\Phi}{a_{1} a_{5}-2 a_{4} x_{2 r} x_{1 r}}, \frac{-b-\sqrt{b^{2}-4 a c}}{2 a}\right\} \text {. }
$$

Proof. Studied system (1) has the following characteristic equation at its equilibrium point:

$$
\begin{aligned}
& \Delta(\lambda)=\left|\begin{array}{ccc}
\lambda+a_{1} & -a_{1}-x_{3 r} & -x_{2 r} \\
a_{3} x_{3 r} & \lambda-\left(a_{2}+k\right) & a_{3} x_{1 r} \\
-2 a_{4} x_{1 r} & 0 & \lambda+a_{5}
\end{array}\right|=0, \\
& \Delta(\lambda)=\left|\begin{array}{ccc}
\lambda+a_{1} & -a_{1}-x_{3 r} & -x_{2 r} \\
a_{3} x_{3 r} & \lambda-\left(a_{2}+k\right) & a_{3} x_{1 r} \\
-2 a_{4} x_{1 r} & 0 & \lambda+a_{5}
\end{array}\right|=0,
\end{aligned}
$$

Let $\beta_{2}=\left(a_{1}-k-a_{2}+a_{5}\right), \beta_{1}=-\left(a_{1}+a_{5}\right) k+\Omega$, where $\Omega=a_{3} x_{3 r}^{2}+a_{1} a_{3} x_{3 r}-a_{1} a_{2}+a_{1} a_{5}-a_{2} a_{5}-2 a_{4} x_{1 r} x_{2 r}$ and

$$
\begin{aligned}
\beta_{0} & =a_{3} a_{5} x_{3 r}^{2}-k a_{1} a_{5}-a_{1} a_{2} a_{5}+2 a_{3} a_{4} x_{3 r} x_{1 r}^{2}+2 k a_{4} x_{2 r} x_{1 r}+a_{1} a_{3} a_{5} x_{3 r}+2 a_{1} a_{3} a_{4} x_{1 r}^{2}+2 a_{2} a_{4} x_{2 r} x_{1 r} \\
& =k\left(2 a_{4} x_{2 r} x_{1 r}-a_{1} a_{5}\right)+\Phi
\end{aligned}
$$

where $\Phi=a_{3} a_{5} x_{3 r}^{2}-a_{1} a_{2} a_{5}+2 a_{3} a_{4} x_{3 r} x_{1 r}^{2}+a_{1} a_{3} a_{5} x_{3 r}$ $+2 a_{1} a_{3} a_{4}$.

Then, $\Delta(\lambda)$ can be written as

$$
\lambda^{3}+\beta_{2} \lambda^{2}+\beta_{1} \lambda+\beta_{0}=0
$$

Applying Routh-Hurwitz stability criterion, the eigenvalues of (31) have negative real parts if and only if $\beta_{2}>0$, $\beta_{0}>0$, and $\beta_{2} \beta_{1}-\beta_{0}>0$.
From $\beta_{2}>0$ and $\beta_{0}>0$, it is clear that

$$
\left\{\begin{array}{l}
k<\left(a_{1}-a_{2}+a_{5}\right), \text { and } \\
k<\frac{\Phi}{a_{1} a_{5}-2 a_{4} x_{2 r} x_{1 r}} .
\end{array}\right.
$$

Note that

$$
\begin{aligned}
\beta_{2} \beta_{1}-\beta_{0}= & \left(a_{1}-k-a_{2}+a_{5}\right)\left(-\left(a_{1}+a_{5}\right) k+\Omega\right)-\left(k\left(2 a_{4} x_{2 r} x_{1 r}-a_{1} a_{5}\right)+\Phi\right), \\
= & \left(a_{1}-k-a_{2}+a_{5}\right)\left(-\left(a_{1}+a_{5}\right) k+\Omega\right)-\left(k\left(2 a_{4} x_{2 r} x_{1 r}-a_{1} a_{5}\right)+\Phi\right) \\
= & \left(a_{1}+a_{5}\right) k^{2}+k\left(-\Omega-a_{1}^{2}-a_{5}^{2}+a_{1} a_{2}-a_{1} a_{5}+a_{2} a_{5}-2 a_{4} x_{1 r} x_{2 r}\right) \\
& +\left(\Omega a_{5}+\Omega a_{1}-\Phi-\Omega a_{2}\right) \\
= & a k^{2}+b k+c,
\end{aligned}
$$




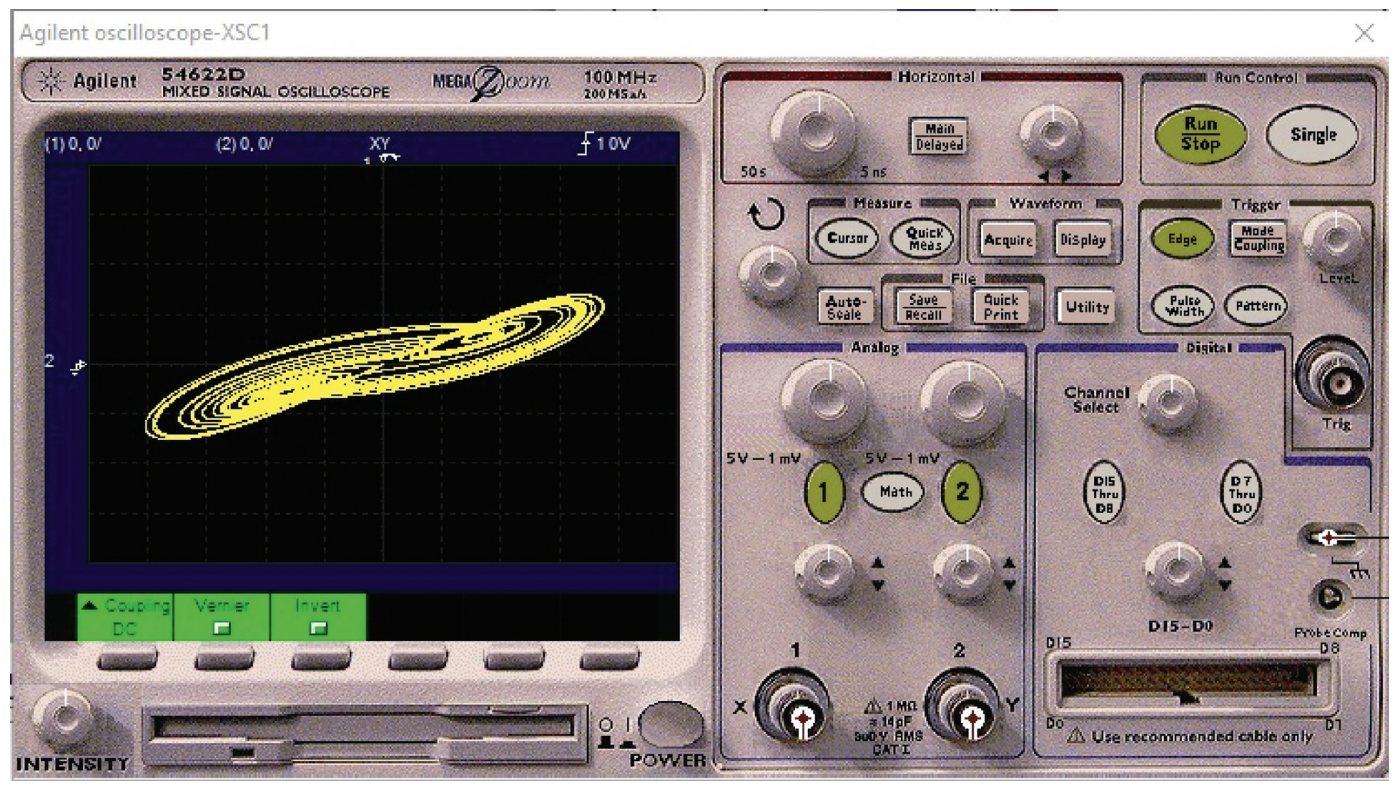

Figure 6: Phase portrait of the state variable $x_{2}$ versus the state variable $x_{1}$.

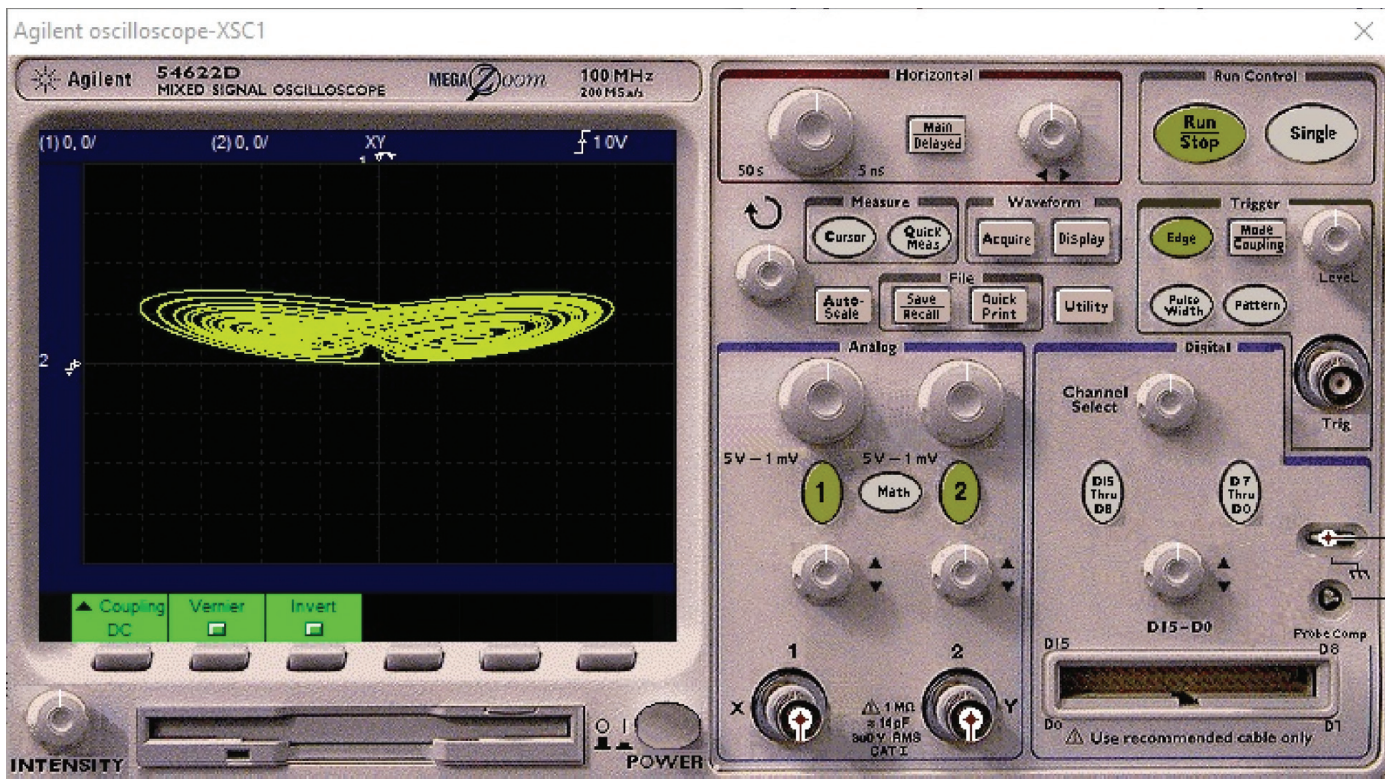

Figure 7: Phase portrait of the state variable $x_{3}$ with respect to the state variable $x_{1}$.

where $\quad a=\left(a_{1}+a_{5}\right), \quad b=\left(-\Omega-a_{1}^{2}-a_{5}^{2}+a_{1} a_{2}-a_{1} a_{5}\right.$ $\left.+a_{2} a_{5}-2 a_{4} x_{1 r} x_{2 r}\right)$, and $c=\left(\Omega a_{5}+\Omega a_{1}-\Phi-\Omega a_{2}\right)$.

From (33) and $\beta_{2} \beta_{1}-\beta_{0}>0$, we can derive that

$$
\left(k-\frac{-b+\sqrt{b^{2}-4 a c}}{2 a}\right)\left(k-\frac{-b-\sqrt{b^{2}-4 a c}}{2 a}\right)>0,
$$

which implies that

$$
k\left\langle\frac{-b-\sqrt{b^{2}-4 a c}}{2 a}, \text { or } k>\frac{-b+\sqrt{b^{2}-4 a c}}{2 a}\right\rangle 0 .
$$

While concluding, to meet $\beta_{2}>0, \quad \beta_{0}>0$, and $\beta_{2} \beta_{1}-\beta_{0}>0$, the control gain can be selected as $k<\min \left\{\left(a_{1}-a_{2}+a_{5}\right), \frac{\Phi}{a_{1} a_{5}-2 a_{4} x_{2 r} x_{1 r}}, \frac{-b-\sqrt{b^{2}-4 a c}}{2 a}\right\}$,

which completes the proof.

5.2. Case Studies of Reference Value-Based Control of the Studied System

Case 1. $x_{1 r}=x_{2 r}=x_{3 r}=0$

Applying Theorem 1, the controller gain can be calculated and we can select it $k=-8$. Proposed controller (24) is 


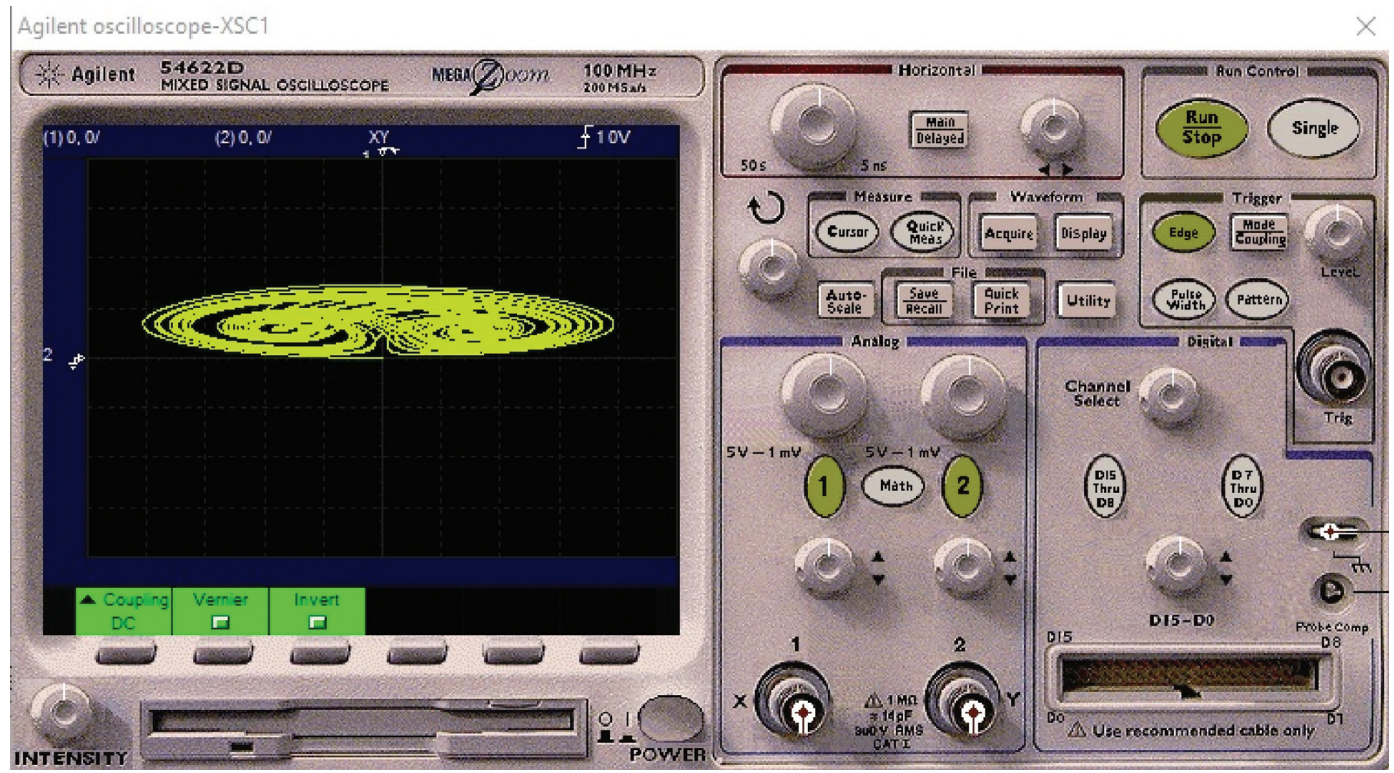

FIgURE 8: Phase portrait of the state variable $x_{3}$ with respect to the state variable $x_{2}$.

TABle 2: The input/output gains of the multipliers.

\begin{tabular}{lccc}
\hline & Output gain & X gain & Y gain \\
\hline S1 & 1 & 1 & 1 \\
S2 & 1 & 1 & 1 \\
A3 & 1 & 1 & 1 \\
\hline
\end{tabular}

applied to studied system (1) at $t=30$ secs. Figure 9 shows the system states before and after applying control. It is obvious that the proposed controller drives the state variables to follow their reference values after applying it immediately.

Case 2. $x_{1 r}=10, x_{2 r}=-10, x_{3 r}=20$

Applying Theorem 1, the controller gain can be calculated and we can select it $k=-50$. Proposed controller (24) is applied to studied system (1) at $t=30$ secs. Figure 10 shows the system states before and after applying control. It is obvious that the proposed controller drives the state variables to follow their reference values after applying it immediately.

Case 3. $x_{1 r}=-6, x_{2 r}=5, x_{3 r}=-6 \sqrt{2}$

Applying Theorem 1, the controller gain can be calculated and we can select it $k=-60$. Proposed controller (24) is applied to studied system (1) at $t=30 \mathrm{secs}$. Figure 11 shows the system states before and after applying control. It is obvious that the proposed controller drives the state variables to follow their reference values after applying it immediately.

\section{Robust Controller}

In this section, a tracking robust controller is to be designed. Since in practice, the uncertainties are unavoidable matter, then it is important to design a robust single-state feedback tracking controller for the studied system.

6.1. Robust Track Controller Algorithm Design. Suppose the system parameters have some perturbations. The fuzzy studied system can be written in the following format:

$$
\begin{aligned}
& \left(\frac{\mathrm{d} x_{1}}{\mathrm{~d} t}\right)=\left(a_{1}+\Delta a_{1}\right) x_{2}-\left(a_{1}+\Delta a_{1}\right) x_{1}+x_{2} x_{3}+C_{1}, \\
& \left(\frac{\mathrm{d} x_{2}}{\mathrm{~d} t}\right)=\left(a_{2}+\Delta a_{2}\right) x_{2}-\left(a_{3}+\Delta a_{3}\right) x_{1} x_{3}+C_{2}, \\
& \left(\frac{\mathrm{d} x_{3}}{\mathrm{~d} t}\right)=\left(a_{4}+\Delta a_{4}\right) x_{1}^{2}-\left(a_{5}+\Delta a_{5}\right) x_{3}+C_{3} .
\end{aligned}
$$

Simplifying (38), we get

$$
\begin{aligned}
& \left(\frac{\mathrm{d} x_{1}}{\mathrm{~d} t}\right)=a_{1} x_{2}-a_{1} x_{1}+x_{2} x_{3}+\Delta a_{1}\left(x_{2}-x_{1}\right)+C_{1} \\
& \left(\frac{\mathrm{d} x_{2}}{\mathrm{~d} t}\right)=a_{2} x_{2}-a_{3} x_{1} x_{3}+\Delta a_{2} x_{2}-\Delta a_{3} x_{1} x_{3}+C_{2} \\
& \left(\frac{\mathrm{d} x_{3}}{\mathrm{~d} t}\right)=a_{4} x_{1}^{2}-a_{5} x_{3}+\Delta a_{4} x_{1}^{2}-\Delta a_{5} x_{3}+C_{3} .
\end{aligned}
$$

The uncertainties are collected in a column vector $u=$ $\left(u_{1}, u_{2}, u_{3}\right)^{T} \quad$ where $\quad u_{1}=\Delta a_{1}\left(x_{2}-x_{1}\right), \quad u_{2}=\Delta a_{2} x_{2}$ $-\Delta a_{3} x_{1} x_{3}$, and $u_{3}=\Delta a_{4} x_{1}^{2}-\Delta a_{5} x_{3}$. Applying designed controller (24) upon system (39), the new dynamics of the tracking error will be as

$$
\begin{aligned}
& \left(\frac{\mathrm{d} E_{1}}{\mathrm{~d} t}\right)=a_{1} E_{2}-a_{1} E_{1}+E_{2} E_{3}+x_{3 r} E_{2}+x_{2 r} E_{3}+u_{1}, \\
& \left(\frac{\mathrm{d} E_{2}}{\mathrm{~d} t}\right)=\left(a_{2}+k\right) E_{2}-a_{3} E_{1} E_{3}-a_{3} x_{3 r} E_{1}-a_{3} x_{1 r} E_{3}+u_{2}, \\
& \left(\frac{\mathrm{d} E_{3}}{\mathrm{~d} t}\right)=a_{4} E_{1}^{2}+2 a_{4} x_{1 r} E_{1}-a_{5} E_{3}+u_{3} .
\end{aligned}
$$




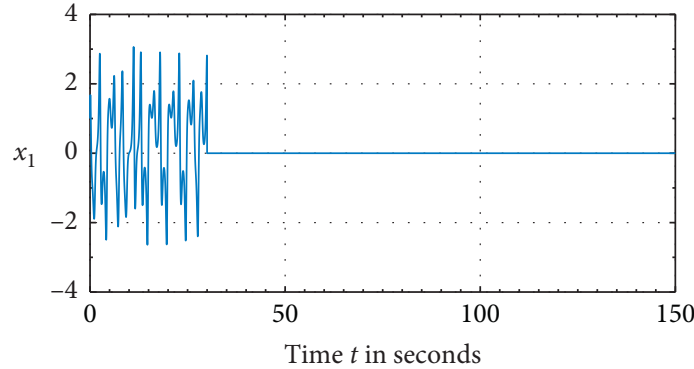

(a)

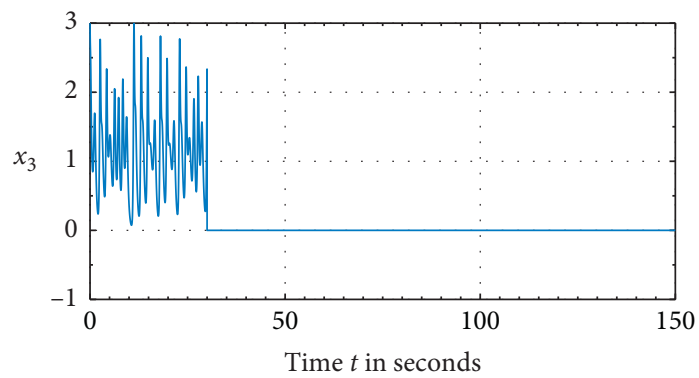

(c)

(c)

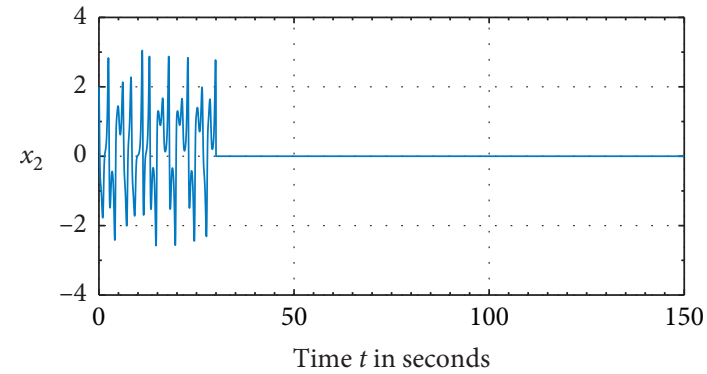

(b)

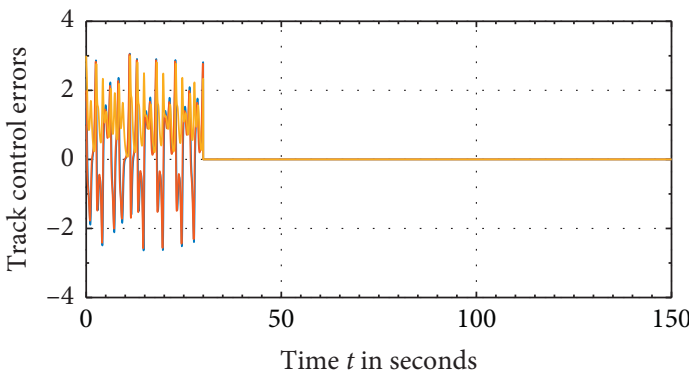

$-E_{1}$
$-E_{2}$
$E_{3}$

(d)

Figure 9: State dynamics under the track control method with reference $(0,0,0)$ and the controller gain $k=-8$, where the controller is applied after 30 secs. (a) The state variable $x_{1}$; (b) the state variable $x_{2}$; (c) the state variable $x_{3}$; (d) track control errors $E_{1}$, $E_{2}$, and $E_{3}$.

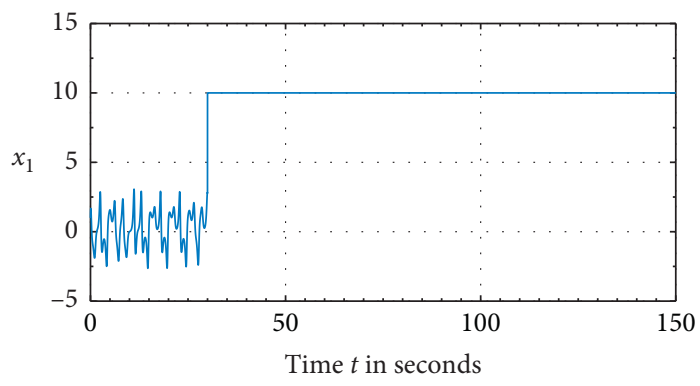

(a)

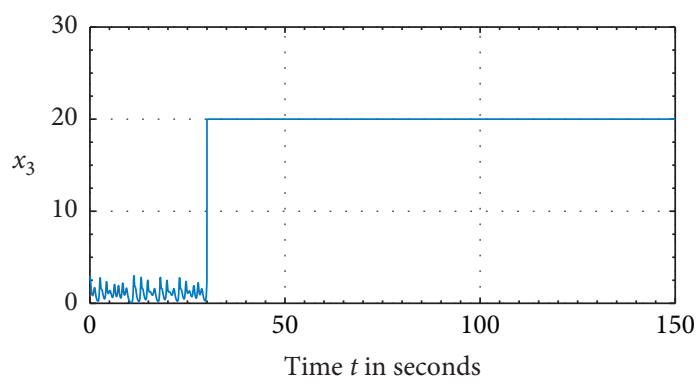

(c)

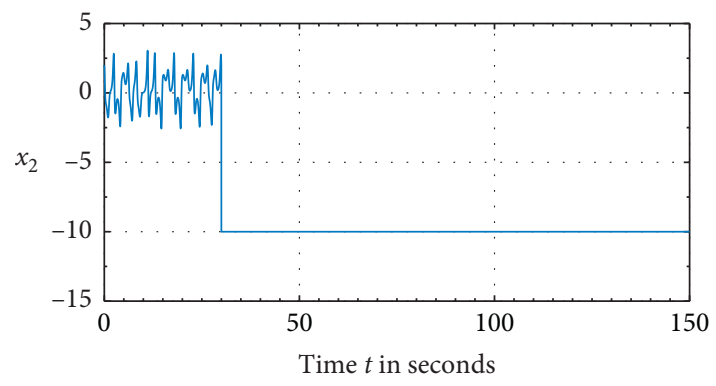

(b)

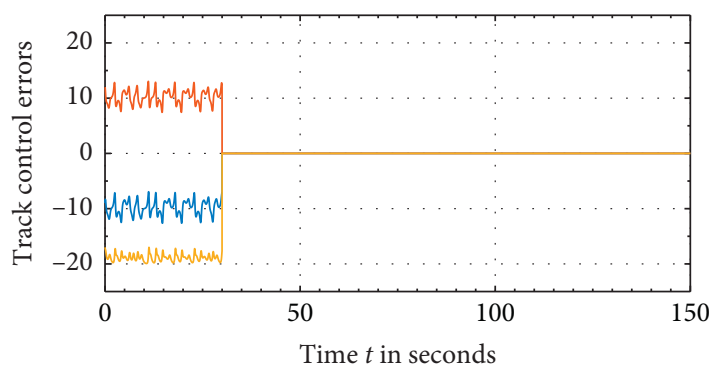

$$
\begin{gathered}
E_{1} \\
-E_{2} \\
-E_{3}
\end{gathered}
$$

(d)

Figure 10: State dynamics under the track control method with reference $(10,-10,20)$ and the controller gain $k=-50$, where the controller is applied after 30 secs. (a) The state variable $x_{1}$; (b) the state variable $x_{2}$; (c) the state variable $x_{3}$; (d) track control errors $E_{1}$, $E_{2}$, and $E_{3}$. 


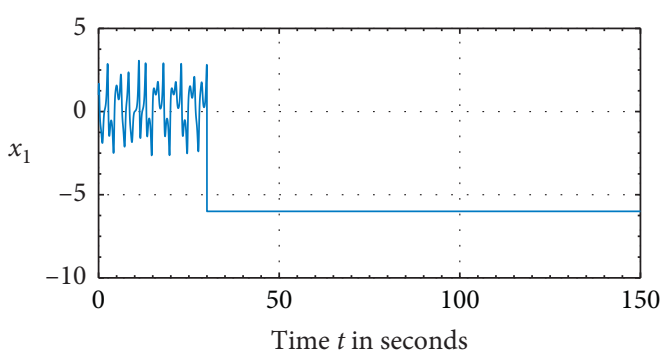

(a)

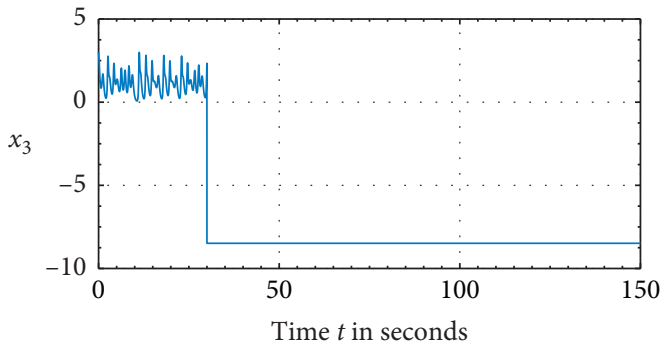

(c)

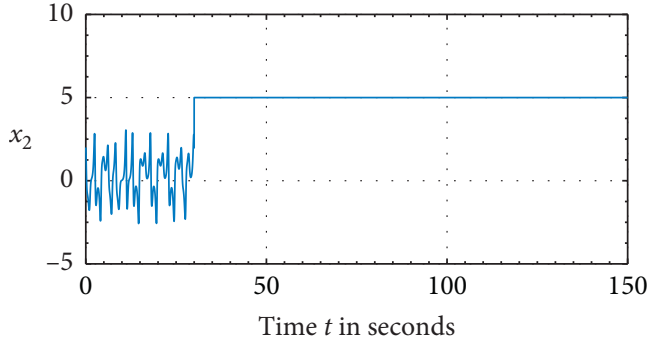

(b)

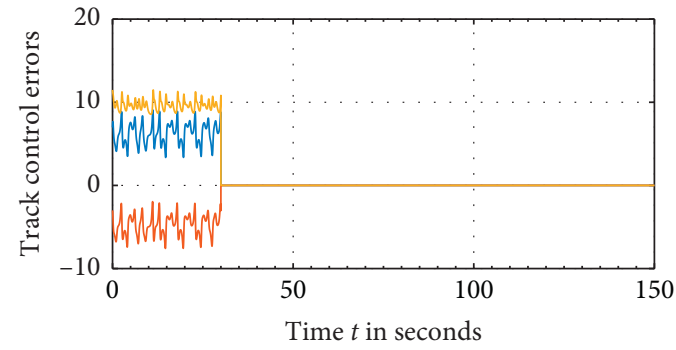

$-E_{1}$
$-E_{2}$
$-E_{3}$

FIgURE 11: State dynamics under the track control method with reference $(10,-10,20)$ and the controller gain $k=-50$, where the controller is applied after 30 secs. (a) The state variable $x_{1}$; (b) the state variable $x_{2}$; (c) the state variable $x_{3}$; (d) track control errors $E_{1}$, $E_{2}$, and $E_{3}$.

That can be written as

$$
\left[\begin{array}{l}
\left(\frac{\mathrm{d} E_{1}}{\mathrm{~d} t}\right) \\
\left(\frac{\mathrm{d} E_{2}}{\mathrm{~d} t}\right) \\
\left(\frac{\mathrm{d} E_{3}}{\mathrm{~d} t}\right)
\end{array}\right]=\left[\begin{array}{ccc}
-a_{1} & \left(a_{1}+x_{3 r}\right) & x_{2 r} \\
-a_{3} x_{3 r} & \left(a_{2}+k\right) & -a_{3} x_{1 r} \\
2 a_{4} x_{1 r} & 0 & -a_{5}
\end{array}\right]\left[\begin{array}{c}
E_{1} \\
E_{2} \\
E_{3}
\end{array}\right]+\left[\begin{array}{c}
E_{2} E_{3}+u_{1} \\
-a_{3} E_{1} E_{3}+u_{2} \\
a_{4} E_{1}^{2}+u_{3}
\end{array}\right]
$$

Let

$$
\begin{aligned}
E & =\left[\begin{array}{l}
E_{1} \\
E_{2} \\
E_{3}
\end{array}\right], \\
A & =\left[\begin{array}{ccc}
-a_{1} & \left(a_{1}+x_{3 r}\right) & x_{2 r} \\
-a_{3} x_{3 r} & \left(a_{2}+k\right) & -a_{3} x_{1 r} \\
2 a_{4} x_{1 r} & 0 & -a_{5}
\end{array}\right], \\
U & =\left[\begin{array}{c}
U_{1} \\
U_{2} \\
U_{3}
\end{array}\right]=\left[\begin{array}{c}
E_{2} E_{3}+u_{1} \\
-a_{3} E_{1} E_{3}+u_{2} \\
a_{4} E_{1}^{2}+u_{3}
\end{array}\right] .
\end{aligned}
$$

Then, the Laplace transformation of the track control error system $\dot{E}=A E+U$ is given as follows: $E(s)=(s I-A)^{-1} U(s)$.

Since the controller gain has no effect upon the uncertain terms $U_{1}$ and $U_{3}$, an improvement to the single-state feedback controller is designed as follows:

$$
\begin{aligned}
& C_{1}=k_{1}\left(x_{2}-x_{2 r}\right)-a_{1} x_{2 r}+a_{1} x_{1 r}-x_{2 r} x_{3 r}, \\
& C_{2}=k_{2}\left(x_{2}-x_{2 r}\right)-a_{2} x_{2 r}+a_{3} x_{1 r} x_{3 r}, \\
& C_{3}=k_{3}\left(x_{2}-x_{2 r}\right)-a_{4} x_{1 r}^{2}+a_{5} x_{3 r} .
\end{aligned}
$$

Utilizing proposed robust controller (43) with disturbed system (39), the closed-loop track control error dynamics are given as 


$$
\begin{aligned}
& \left(\frac{\mathrm{d} E_{1}}{\mathrm{~d} t}\right)=k_{1} E_{2}+a_{1} E_{2}-a_{1} E_{1}+E_{2} E_{3}+x_{3 r} E_{2}+x_{2 r} E_{3}+u_{1}, \\
& \left(\frac{\mathrm{d} E_{2}}{\mathrm{~d} t}\right)=\left(k_{2}+a_{2}\right) E_{2}-a_{3} E_{1} E_{3}-a_{3} x_{3 r} E_{1}-a_{3} x_{1 r} E_{3}+u_{2}, \\
& \left(\frac{\mathrm{d} E_{3}}{\mathrm{~d} t}\right)=k_{3} E_{2}+a_{4} E_{1}^{2}+2 a_{4} x_{1 r} E_{1}-a_{5} E_{3}+u_{3} .
\end{aligned}
$$

That can be rewritten in a compact format as $\dot{E}=B E+U$ which equal the system

$$
\left[\begin{array}{l}
\left(\frac{\mathrm{d} E_{1}}{\mathrm{~d} t}\right) \\
\left(\frac{\mathrm{d} E_{2}}{\mathrm{~d} t}\right) \\
\left(\frac{\mathrm{d} E_{3}}{\mathrm{~d} t}\right)
\end{array}\right]=\left[\begin{array}{ccc}
-a_{1} & \left(k_{1}+a_{1}+x_{3 r}\right) & x_{2 r} \\
-a_{3} x_{3 r} & \left(a_{2}+k_{2}\right) & -a_{3} x_{1 r} \\
2 a_{4} x_{1 r} & k_{3} & -a_{5}
\end{array}\right]\left[\begin{array}{c}
E_{1} \\
E_{2} \\
E_{3}
\end{array}\right]+\left[\begin{array}{c}
E_{2} E_{3}+u_{1} \\
-a_{3} E_{1} E_{3}+u_{2} \\
a_{4} E_{1}^{2}+u_{3}
\end{array}\right]
$$

Then, the Laplace transform of (45) is got by $E(s)=(s I-B)^{-1} U(s)$.

Consequently, our aim now is to find the values of the gains $k_{1}, k_{2}, k_{3}$ in order to reduce the disturbances effects. To accomplish this task, the following fitness function is to be minimized:

$$
f=\sum_{i}\left\|\left(s_{i} I-B\right)^{-1}\right\|_{s_{i}=j \omega_{d i}},
$$

where $\omega_{d i}=2 \pi f_{i}(i=1,2, \ldots$,$) are the dominant fre-$ quencies related to the disturbances. We can calculate the dominant frequencies using the known fast Fourier transform (FFT) method [33].

Genetic algorithm [34] will be used here to minimize the fitness function (46).

The following is the process of finding the robust controller gains $k_{1}, k_{2}$, and $k_{3}$.

Algorithm of seeking the robust controller gains $k_{1}, k_{2}$, and $k_{3}$ is as follows:

(1) Determine the dominant frequencies for the feedback state variable $x_{2}$

(2) Construct fitness function (46)

(3) The nonlinear constraint is set as $\operatorname{Re}\left(\lambda_{r}\right)+\theta \leq 0$, where for $r=1,2,3, \lambda_{r}$ are the eigenvalues of the matrix and $\theta$ is to be set as a positive real value

(4) Utilize the GA toolbox in Matlab to choose the optimal robust controller gains $k_{1}, k_{2}$, and $k_{3}$
6.2. Numerical Simulation for the Robust Track Controller. In the following simulations, the disturbances are taken as

$$
\begin{aligned}
& u_{1}=\sin (2 \pi t)\left(x_{2}-x_{1}\right)+\text { noise } \\
& u_{2}=\sin (4 \pi t) x_{1}+\text { noise } \\
& u_{3}=\sin (6 \pi t) x_{3}
\end{aligned}
$$

where noise $=n *($ random_number $), n=s /\left(10^{(\mathrm{snr} / 20)}\right)$, snr is the signal to noise ratio that set as $s n r=20 \log (s / n)=100$, and $s$ is the standard deviation of $x_{2}$.

For illustration, the references are selected to be $x_{1 r}=-20, x_{2 r}=40$, and $x_{3 r}=-60$. Using FFT, the dominant frequencies of $x_{2}$ are selected as $f_{1}=0.206653 \mathrm{HZ}$; $f_{2}=0.319979 \mathrm{HZ} ; \quad f_{3}=0.333311 \mathrm{HZ} ; f_{4}=0.786614 \mathrm{HZ}$; $f_{5}=0.293313779 \mathrm{HZ} ; \quad f_{6}=0.213319112 \mathrm{HZ} ; \quad f_{7}=$ $0.166655556 ; f_{8}=0.193320445 ;$ and $f_{9}=0.219985334$. Figure 12 displays the spectrum of $x_{2}$ using the FFT method. The selected dominant frequencies are used to construct fitness function (46). $\theta$ is set to equal 3 . Figure 13 shows the optimization process via GA. The selected gains are $k_{1}=-116.67 ; k_{2}=-332 ; k_{3}=-68$. Figure 14 shows the system response before and after control with the corresponding track errors. The controller drives the states to follow the reference values: $x_{1 r}=x_{2 r}=x_{3 r}=0$. Figure 15 shows the system response before and after control with the corresponding track errors. The controller drives the states to follow the reference values: $x_{1 r}=-5 ; x_{2 r}=5 ; x_{3 r}=-6$. 


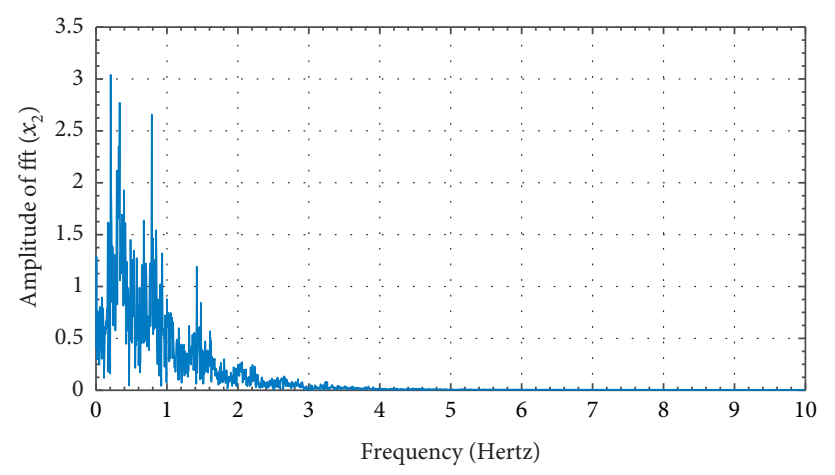

Figure 12: Amplitude versus frequencies of $x_{2}$ spectrum via FFT.

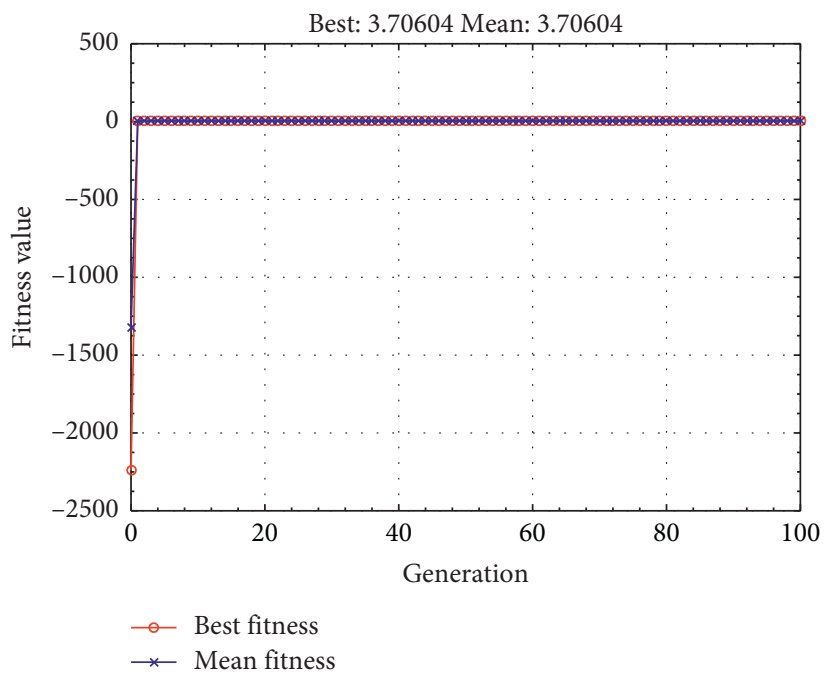

FIGURE 13: Fitness value versus generations in GA optimization process.

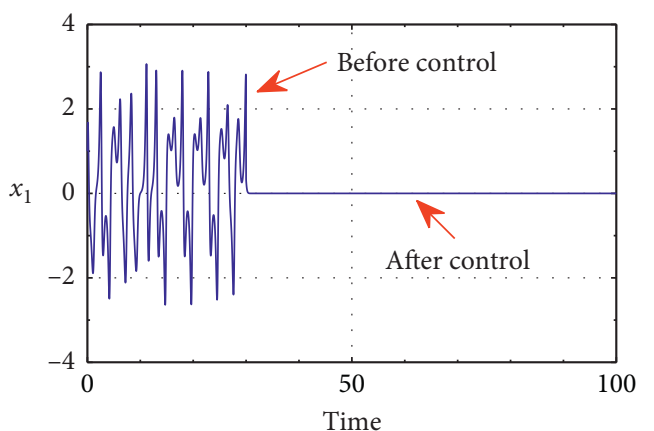

(a)

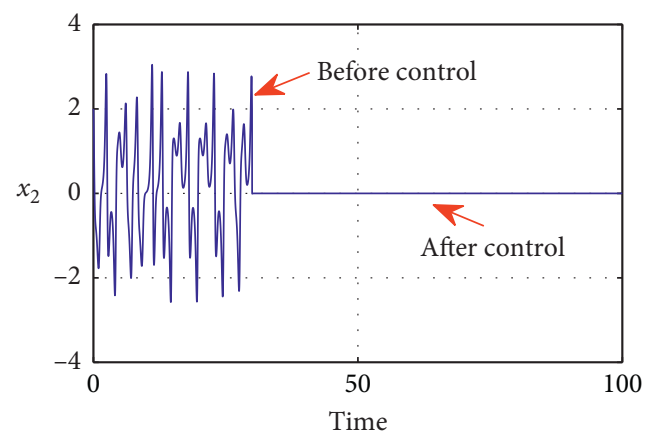

(b)

Figure 14: Continued. 


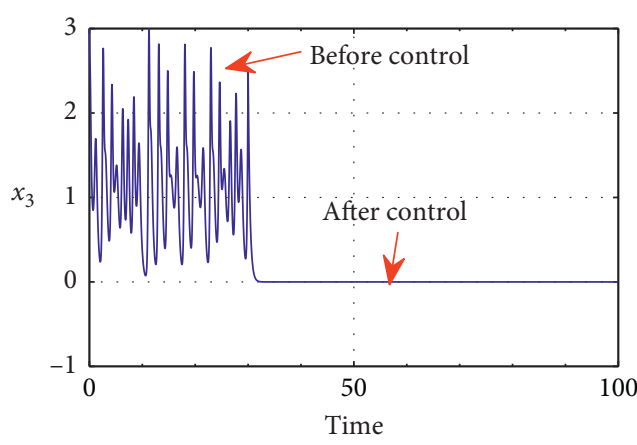

(c)

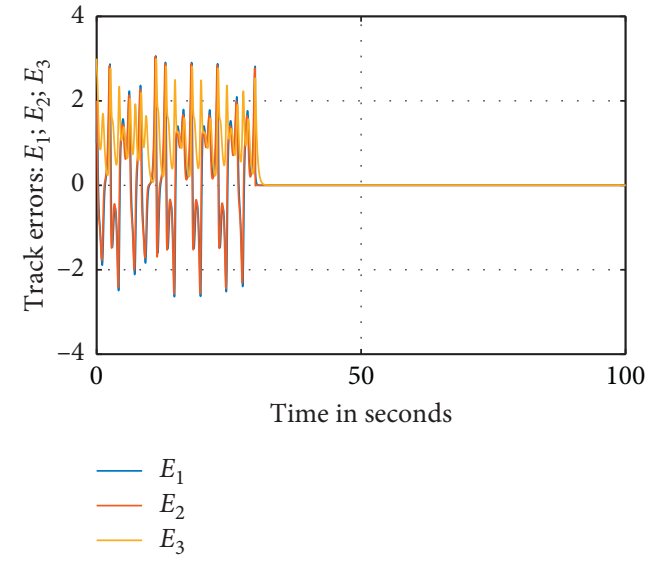

(d)

FiguRe 14: The system response before and after control with $x_{1 r}=x_{2 r}=x_{3 r}=0$ : (a) the state variable $x_{1}$ before and after control; (b) state variable $x_{2}$ before and after control; (c) the state variable $x_{3}$ before and after control; (d) the track errors before and after control.

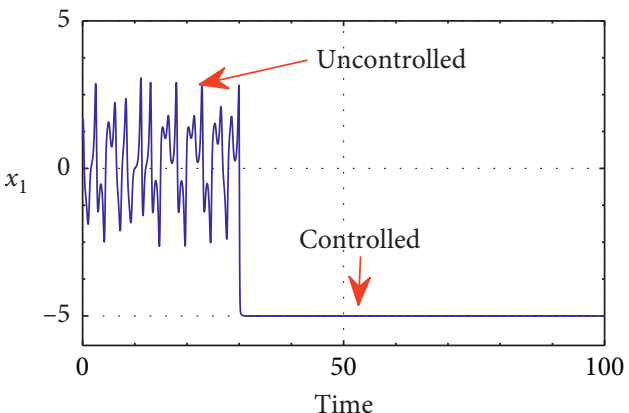

(a)

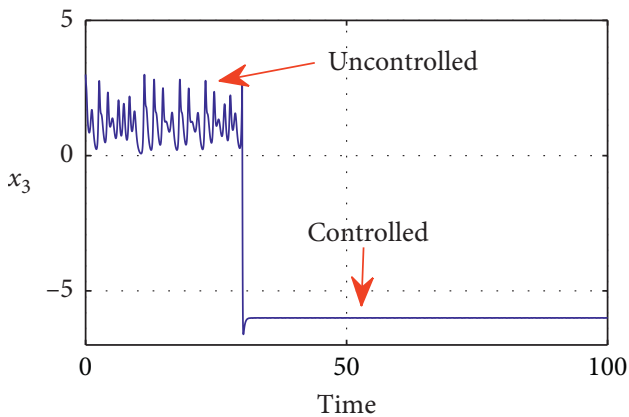

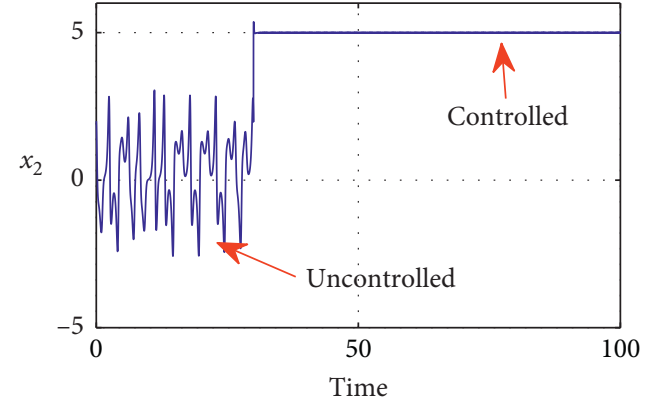

(b)

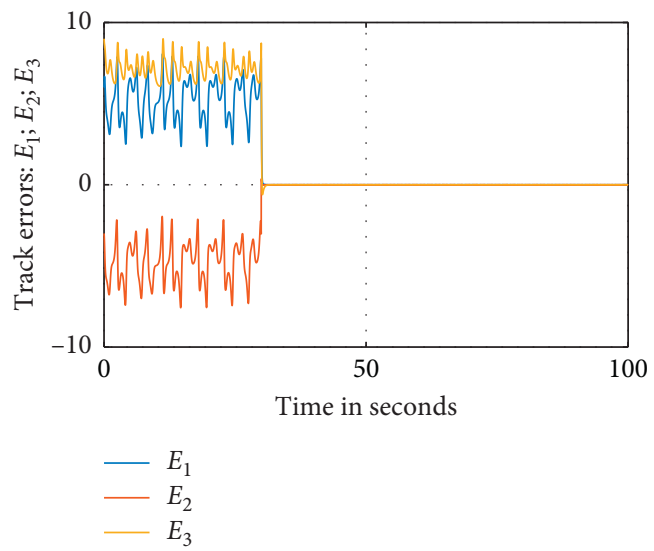

(d)

Figure 15: The system response before and after control with $x_{1 r}=-5 ; x_{2 r}=5 ; x_{3 r}=-6$ : (a) the state variable $x_{1}$ before and after control; (b) the state variable $x_{2}$ before and after control; (c) the state variable $x_{3}$ before and after control; (d) track errors before and after control.

\section{Conclusion}

In this work, the dynamics of a new proposed chaotic model have been studied. The new proposed model chaotic behaviors have been proved by calculating its Lyapunov exponents. The proposed model has been studied via discussing its symmetry and invariance, dissipativity, and the stability of its fixed point plus its Lyapunov exponents. The proposed model has been confirmed to be chaotic. The proposed chaotic model complexity has been measured using its SFG. The proposed chaotic model has been confirmed to be realizable via constructing areal electronic circuit that simulate its behavior. In addition, a new singlestate feedback controller has been designed in order to 
operate the system such that its states can follow a predetermined reference value. The robustness of the proposed controller has been discussed. The proposed robust controller has been constructed with the aid of the genetic algorithm. For future work, we suggest utilizing the proposed model in real applications such as secure communication applications.

\section{Data Availability}

No data were used to support the findings of this study.

\section{Conflicts of Interest}

The authors have no conflicts of interest regarding the publication of the paper.

\section{Acknowledgments}

This research was funded by the Deanship of Scientific Research, Taif University, KSA (research group number 11441-105).

\section{References}

[1] C. Sparrow, The Lorenz Equations: Bifurcations, Chaos and Strange Attractors, Springer, New York, NY, USA, 1982.

[2] O. E. Rössler, "An equation for continuous chaos," Physics Letters A, vol. 57, no. 5, pp. 397-398, 1976.

[3] G. Chen and T. Ueta, "Yet another chaotic attractor," International Journal of Bifurcation and Chaos, vol. 9, no. 7, pp. 1465-1466, 1999.

[4] J. Lü and G. Chen, "A new chaotic attractor coined," International Journal of Bifurcation and Chaos, vol. 12, no. 3, pp. 659-661, 2002.

[5] J. Lü, G. Chen, D. Cheng, and S. Celikovsky, "Bridge the gap between the Lorenz system and the Chen system," International Journal of Bifurcation and Chaos, vol. 12, no. 12, pp. 2917-2926, 2002.

[6] G. Qi, G. Chen, S. Du, Z. Chen, and Z. Yuan, "Analysis of a new chaotic system," Physica A: Statistical Mechanics and its Applications, vol. 352, no. 2-4, pp. 295-308, 2005.

[7] G. Qi, Z. Chen, and Z. Yuan, "Model-free control of affine chaotic systems," Physics Letters A, vol. 344, no. 2-4, pp. 189-202, 2005.

[8] B. R. Nana Nbendjo and P. Woafo, "Active control with delay of horseshoes chaos using piezoelectric absorber on a buckled beam under parametric excitation," Chaos, Solitons \& Fractals, vol. 32, pp. 73-79, 2007.

[9] J. H. Park, "Chaos synchronization between two different chaotic dynamical systems," Chaos, Solitons \& Fractals, vol. 27, no. 2, pp. 549-554, 2006.

[10] C. Wu, T. Fang, and H. Rong, "Chaos synchronization of two stochastic duffing oscillators by feedback control," Chaos, Solitons \& Fractals, vol. 32, no. 3, pp. 1201-1207, 2007.

[11] S. Wiggins, Introduction to Applied Non-Linear Dynamical Systems and Chaos, Springer-Verlag, New York, NY, USA, 1990.

[12] F. Zhang and H. Jack, "Non-chaotic behavior in three-dimensional quadratic systems," Non-linearity, vol. 10, pp. 1289-1303, 1997.

[13] J. C. Sprott, "Simplest dissipative chaotic flow," Physics Letters A, vol. 228, no. 4-5, pp. 271-274, 1997.
[14] F. C. Moon, Chaotic and Fractal Dynamics, Wiley, New York, NY, USA, 1992.

[15] H. K. Khalil, Nonlinear System, Macmillan Publishing Company, New York, NY, USA, 1992.

[16] E. E. Mahmoud and G. M. Mahmoud, On Some Chaotic Complex Nonlinear Systems, Lambert Academic Publishing, Saarbrücken, Germany, 2010.

[17] E. E. Mahmoud and G. M. Mahmoud, Chaotic and Hyperchaotic Nonlinear Systems, Lambert Academic Publishing, Saarbrücken, Germany, 2011.

[18] D. S. Eppinger and R. T. Browning, Design Structure Matrix Methods and Applications, MIT Press, Cambridge, MA, USA, 2012.

[19] I. Mezit, A. Vladimir, M. F. Fonoberov, and T. Sahai, "Spectral complexity of directed graphs and application to structural decomposition," Complexity, vol. 2019, Article ID 9610826, 18 pages, 2019.

[20] K. J. Aström and R. M. Murray, Feedback Systems: An Introduction for Scientists and Engineers, Princeton University Press, Princeton, NJ, USA, 2010.

[21] S.-K. Yang, C.-L. Chen, and H.-T. Yau, "Control of chaos in Lorenz system," Chaos, Solitons \& Fractals, vol. 13, no. 4, pp. 767-780, 2002.

[22] R. Gao, "A novel track control for Lorenz system with single state feedback," Chaos, Solitons \& Fractals, vol. 122, pp. 236-244, 2019.

[23] E. E. Mahmoud, M. Higazy, and O. A. Althagafi, "A novel strategy for complete and phase robust synchronizations of chaotic nonlinear systems," Symmetry, vol. 12, no. 11, p. 1765, 2020.

[24] L. Ma, G. Zong, X. Zhao, and X. Huo, "Observed-based adaptive finite-time tracking control for a class of nonstrictfeedback nonlinear systems with input saturation," Journal of the Franklin Institute, vol. 357, no. 16, pp. 11518-11544, 2020.

[25] Y. Chang, S. Zhang, N. D. Alotaibi, and A. F. Alkhateeb, "Observer-based adaptive finite-time tracking control for a class of switched nonlinear systems with unmodeled dynamics," IEEE Access, vol. 8, pp. 204782-204790, 2020.

[26] B. Wu, X.-H. Chang, and X. Zhao, "Fuzzy Ho output feedback control for nonlinear NCSs with quantization and stochastic communication protocol," IEEE Transactions on Fuzzy Systems, p. 1, 2020, In press.

[27] X.-H. Chang, J. Xiong, and J. H. Park, "Fuzzy robust dynamic output feedback control of nonlinear systems with linear fractional parametric uncertainties," Applied Mathematics and Computation, vol. 291, pp. 213-225, 2016.

[28] Y. Wang, Y. Chang, A. Alkhateeb, and N. D. Alotaibi, "Adaptive fuzzy output-feedback tracking control for switched nonstrict-feedback nonlinear systems with prescribed performance," Circuits Systems and Signal Processing, 2020, In press.

[29] A. M. S. Mahdy, M. Higazy, K. A. Gepreel, and A. A. A. ElDahdouh, "Optimal control and bifurcation diagram for a model nonlinear fractional SIRC," Alexandria Engineering Journal, vol. 59, no. 5, pp. 3481-3501, 2020.

[30] A. M. S. Mahdy, N. H. Sweilam, and M. Higazy, "Approximate solution for solving nonlinear fractional order smoking model," Alexandria Engineering Journal, vol. 59, no. 2, pp. 739-752, 2020.

[31] M. Higazy, "Novel fractional order SIDARTHE mathematical model of COVID-19 pandemic," Chaos, Solitons \& Fractals, vol. 138, Article ID 110007, 2020.

[32] M. Higazy and M. A. Alyami, "New Caputo-Fabrizio fractional order SEIASqEqHR model for COVID-19 epidemic 
transmission with genetic algorithm based control strategy," Alexandria Engineering Journal, vol. 59, no. 6, pp. 4719-4736, 2020.

[33] R. Bracewell, The Fourier Transform and its Applications, McGraw-Hill, Boston, MA, USA, 2000.

[34] M. Melanie, An Introduction to Genetic Algorithms, MIT Press, Cambridge, MA, USA, 1996. 